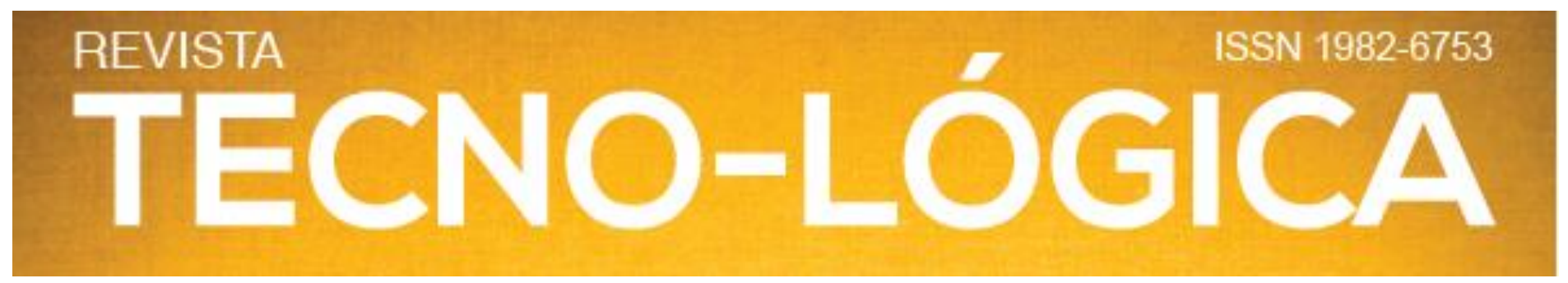

\title{
AVALIAÇÃO ERGONÔMICA DO POSTO DO OPERADOR DE DUAS COLHEDORAS DE GRÃOS
}

\author{
Alexandre Russini ${ }^{l}{ }^{*}$, José Fernando Schlosser ${ }^{2}$, Marcelo Silveira Farias ${ }^{3}$, Daniela Herzog ${ }^{4}$ \\ ${ }^{I}$ Campus Itaqui, Universidade Federal do Pampa, 97650-000, Itaqui, Brasil. \\ ${ }^{2}$ Departamento de Engenharia Rural, Universidade Federal de Santa Maria, 97105-900, Santa Maria, Brasil. \\ ${ }^{3}$ Universidade Federal de Santa Maria, Campus de Frederico Westphalen, 98400-000, Frederico Westphalen, Brasil. \\ ${ }^{4}$ Programa de Pós-Graduação em Engenharia Agrícola, Universidade Federal de Santa Maria, 97105-900, Santa Maria, Brasil.
}

*E-mail: alexandrerussini@unipampa.edu.br

Recebido em: 21/01/2021

Aceito em: 19/06/2021

DOI:10.17058/tecnolog.v25i2.16177

\section{RESUMO}

As condições ergonômicas relacionadas ao posto de operação têm sido determinantes para o desenvolvimento de novos projetos de máquinas agrícolas, proporcionando condições adequadas aos operadores e eficiência durante a realização das operações. Nesse sentido, este trabalho teve como objetivo avaliar as características ergonômicas do posto do operador de duas colhedoras autopropelidas de grãos, relacionadas às zonas de acesso e aos comandos de operação. Foram estudadas duas colhedoras de grãos, denominadas de A e B, pertencentes a Classe IV, sendo avaliado o posicionamento dos principais comandos de operação em relação ao Seat Index Point, classificados em zonas de acesso, conforme a norma ISO 15077. Os resultados indicam que, 70,67\% e 56,75\% dos comandos de acionamento frequente estão localizados nas zonas de conforto e de acesso no plano longitudinal vertical sendo que, em relação ao plano horizontal superior, $100 \%$ e $71,43 \%$ dos comandos encontram-se nestas mesmas zonas para as colhedoras A e B, respectivamente. Conclui-se que, para os dois modelos de colhedoras estudados, ocorreu considerável variação na disposição dos comandos de acionamento para os planos longitudinal vertical e horizontal superior.

Palavras-chave: Mecanização agrícola. Comandos de operação. Ergonomia.

\section{Introdução}

As máquinas agrícolas são essenciais para o agronegócio brasileiro, pois minimizam o esforço dos trabalhadores, melhoram o rendimento e proporcionam qualidade e confiabilidade nas operações de campo [1]. Dentre estas máquinas, destacam-se as colhedoras de grãos que retiram a parte de interesse comercial das plantas. Desta forma, a colheita é considerada a operação mais importante na agricultura, devido ao seu alto valor agregado e, quando executada de maneira adequada, garante o retorno dos investimentos realizados durante o ciclo produtivo da cultura [2].

Conforme IIda [3], ao analisar a atividade do ponto de vista operacional, o posto de operação das colhedoras deve proporcionar aos operadores condições de trabalho que reduzam as restrições biomecânicas e cognitivas, ajudando os operadores a manterem a postura de trabalho adequada, por meio da aplicação de critérios ergonômicos que permitam estabelecer a correta adaptação dos componentes do sistema homem-máquina [4]. Logo, o estudo da adaptação do trabalho ao homem define-se como ergonomia [3]. De acordo com Andrade [5], a ergonomia é a área do conhecimento que tem como objetivo analisar fatores de risco em diversas situações de trabalho, visando propostas de melhorias, unindo o conforto, segurança e a eficiência no trabalho.

Para Rozin et al. [6], os avanços do conhecimento em ergonomia contribuíram para o surgimento de novos conceitos, fazendo com que os fabricantes passassem a ofertar modelos de máquinas agrícolas com melhor disposição dos comandos de operação e instrumentos de controle. Nesse sentido, Barbieri et al. [7] descrevem que o posto de operação é fundamental no desenvolvimento de um novo projeto de máquina, pois é onde se passa a maior parte do tempo durante a realização de determinada operação agrícola. 


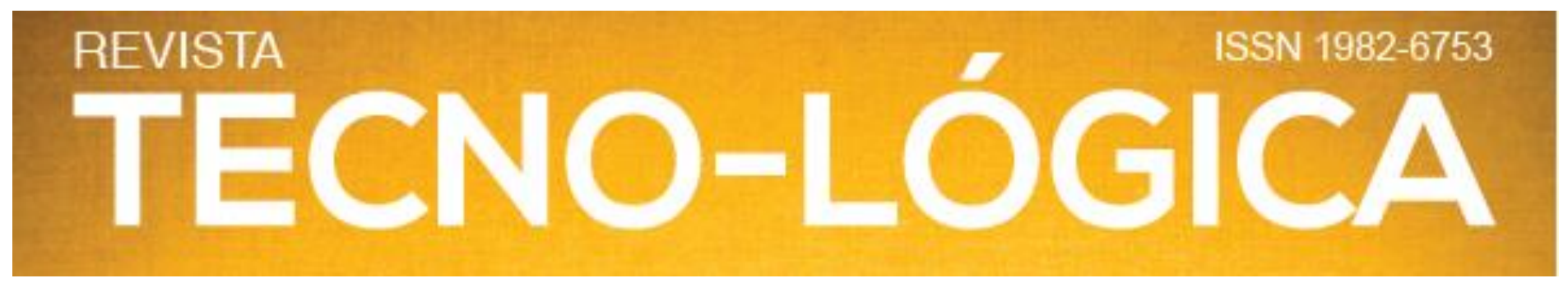

Segundo Dul \& Weerdmeester [8], o uso e o dimensionamento errado dos controles, comandos e manejos, por um longo período, pode provocar lesões, diminuição da produtividade e irritabilidade do operador. Portanto, Casali et al. [9] destacam que as dimensões do posto de operação de uma máquina agrícola devem respeitar o fator humano, seguindo os padrões estabelecidos pelas Normas ISO - International Organization for Standardization, ABNT - Associação Brasileira de Normas Técnicas e ASABE - American Society of Agricultural and Biological Engineers.

No posto de operação, recomenda-se a utilização de distâncias máximas e mínimas que proporcionem conforto ao homem uma vez que, ao não satisfazerem aos parâmetros prédefinidos, dificultam a operação [10]. Ademais, Peripolli et al. [11] mencionam que, quando os espaços internos são respeitados, segundo as normas, proporcionam maior conforto aos operadores e auxiliam na manutenção da saúde e bem-estar, reduzem os riscos de acidentes de trabalho e aumentam o rendimento operacional.

Diante do exposto, este trabalho teve como objetivo avaliar as características ergonômicas do posto do operador de duas colhedoras autopropelidas de grãos, relacionadas às zonas de acesso e aos comandos de operação.

\section{Metodologia}

O trabalho foi realizado por meio da avaliação ergonômica de duas colhedoras autopropelidas de grãos, de diferentes marcas e comumente comercializadas no Brasil. A fim de manter a imparcialidade do estudo e preservar a identidade dos fabricantes, as colhedoras foram denominadas de Colhedora A e Colhedora B, ambas pertencentes a Classe IV, equipadas com motores de 132,48 kW (180 cv) de potência, nas rotações nominais de 2.200 e $2.100 \mathrm{rpm}$, respectivamente.

\subsection{Estabelecimento dos referenciais}

Para referenciar o operador sentado em máquinas agrícolas autopropelidas, adota-se um ponto sobre o assento do operador, denominado de Ponto de Indexação do Assento ou Seat Index Point (SIP), conforme propõe a Norma ISO 5353 [12]. Ainda que, várias normas internacionais façam uso do Ponto de Referência do Assento - Seat Reference Point (SRP) para referenciar o operador sentado, atualmente observa-se a progressiva substituição e adaptação destas para o termo SIP.

Desta forma, cada comando de operação medido nas colhedoras avaliadas foi representado por um ponto identificado por coordenadas em três dimensões, ou seja, três eixos cartesianos (x, y e z), sendo considerados três planos referenciais perpendiculares entre si passando pelo SIP (Figura 1), conforme estabelecido pelas normas ISO 5353 [12], ISO 15077 [13] e ISO 4253 [14].

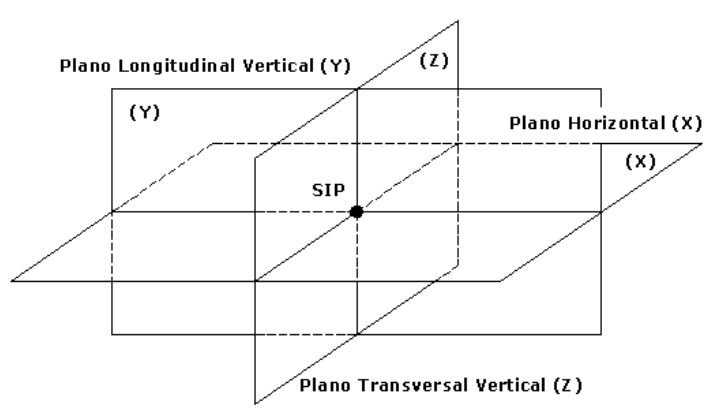

Figura 1 - Planos referenciais perpendiculares entre si passando pelo SIP. Fonte: ISO 15077 [13].

Portanto, o plano longitudinal vertical corta a máquina na sua direção longitudinal, dividindo-a em duas partes: a parte da esquerda e a parte da direita, indicando o posicionamento lateral dos comandos em relação ao SIP. Por sua vez, o plano transversal vertical corta a máquina na direção transversal em referência ao SIP, dividindo-a em duas partes: a anterior e a posterior, indicando o posicionamento frontal e traseiro dos comandos de operação. Da mesma forma, o plano horizontal divide a máquina agrícola em dois hemisférios: o superior e o inferior, estabelecendo a altura dos comandos de operação em relação ao SIP.

\subsection{Coleta de dados}

O procedimento de avaliação ergonômica consistiu na tomada das medidas de coordenadas e dimensões de todos os comandos do posto de operação em relação ao SIP, considerando apenas os planos longitudinal vertical e horizontal superior. Para a determinação do SIP (Figura 2), utilizou-se um dispositivo metálico construído conforme preconiza a norma ISO 5353 [12], seguindo-se os procedimentos para o posicionando deste dispositivo sobre o assento do operador.

Em seguida, foram mensuradas as coordenadas dos principais comandos de mãos e pés nas dimensões definidas, além das principais dimensões ergonômicas do posto de operação, como o assento do operador, o volante de direção e o painel de instrumentos. 


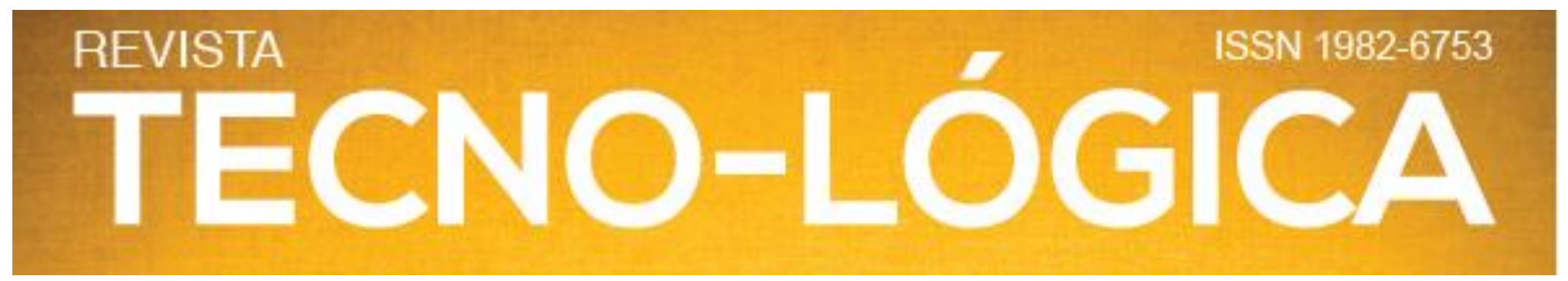

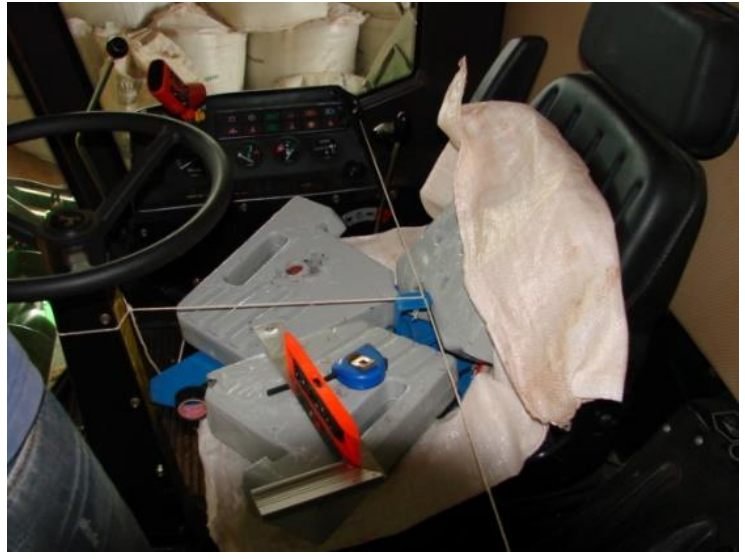

Figura 2 - Dispositivo para determinação do SIP posicionado sobre o assento do operador.

\subsection{Comandos de operação}

A avaliação dos comandos foi realizada considerando os principais comandos básicos de operação, sendo contabilizados 26 e 39 comandos para as colhedoras A e B, respectivamente. Todos os comandos passíveis de movimentação foram colocados em suas posições médias, conforme estabelece a norma ISO 15077 [13]. Para os comandos acionados pela mão, em forma de botão de acionamento mecânico ou eletrônico, foi considerado o ponto de medição que coincide com o centro geométrico da superfície de interface homem/comando.

Para os comandos em forma de alavanca, cuja superfície se aproxima de uma esfera, o ponto de medição corresponde exatamente ao ponto central do comando, localizado na região de interface da mão do operador com o comando. Já para os comandos em forma de alavanca, destinados a aplicação de maiores forças, o ponto de medição corresponde ao ponto central do comando na altura do dedo indicador, representando o ponto de maior esforço de acionamento.

Do mesmo modo, para os comandos de acionamento pelos pés, foi estabelecido um ponto sobre a superfície dos mesmos, resultante da intersecção entre as linhas centro longitudinais e as linhas transversais de cada pedal, conforme preconiza a norma ISO 4253 [14].

\section{4 Áreas de alcance para o operador sentado}

Os principais comandos de operação foram medidos em relação ao plano longitudinal vertical e plano horizontal superior, onde foram obtidas as coordenadas de cada comando em relação ao SIP. As coordenadas dos comandos nas dimensões avaliadas foram confrontadas com as normas ISO 15077 [13] e ISO 4253 [14], que tratam das regiões de alcance do operador sentado e das posições dos comandos de operação.

A norma ISO 15077 [13] estabelece o posicionamento dos comandos internos do posto de operação de acordo com a sua utilização, relacionando-os com os esforços necessários para seu acionamento. Assim, quando um comando é acionado em um intervalo igual ou inferior a cinco minutos, durante o ciclo de funcionamento normal da operação, é classificado como comando de acionamento frequente. Caso esse período for excedido, esse comando é classificado como de acionamento raro.

De acordo com a frequência de utilização dos comandos, a norma ISO 15077 [13] também define áreas ou zonas, que delimitam o posicionamento dos comandos no posto de operação, sendo classificadas como: zonas de conforto, zona de acesso e zonas inacessíveis, classificação válida para comandos de acionamento manual e pelos pés.

A zona de conforto permite fácil acesso aos comandos pelo operador e nela devem estar situados os principais comandos de pé e de mão, estando associada aos comandos frequentemente utilizados. Por sua vez, a zona de acesso permite o alcance do operador desde a sua posição sentado, admitindo deslocamento e inclinação lateral, ou adiante para acionar comandos nessa posição. Associa-se a essa zona os comandos raramente ou pouco utilizados.

Todavia, os comandos localizados na zona inacessível ao alcance do operador ocorrem quando estiver situado fora da zona de conforto ou de acesso, definidas anteriormente. As regiões de alcance do operador sentado são apresentadas no plano longitudinal vertical, bem como no plano horizontal superior, conforme demonstrado na Figura 3.
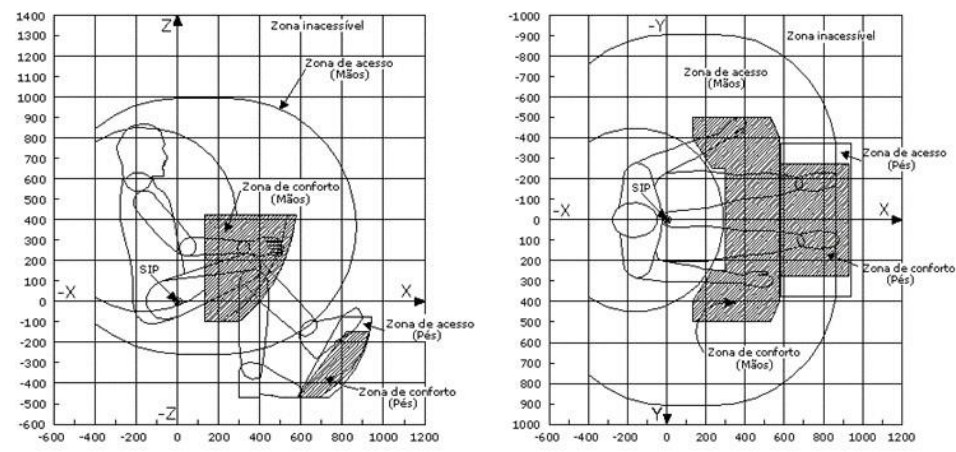

Figura 3 - Regiões de alcance do operador no plano longitudinal vertical (esquerda) e no plano horizontal superior (direita).

Fonte: ISO 15077 [13]. 


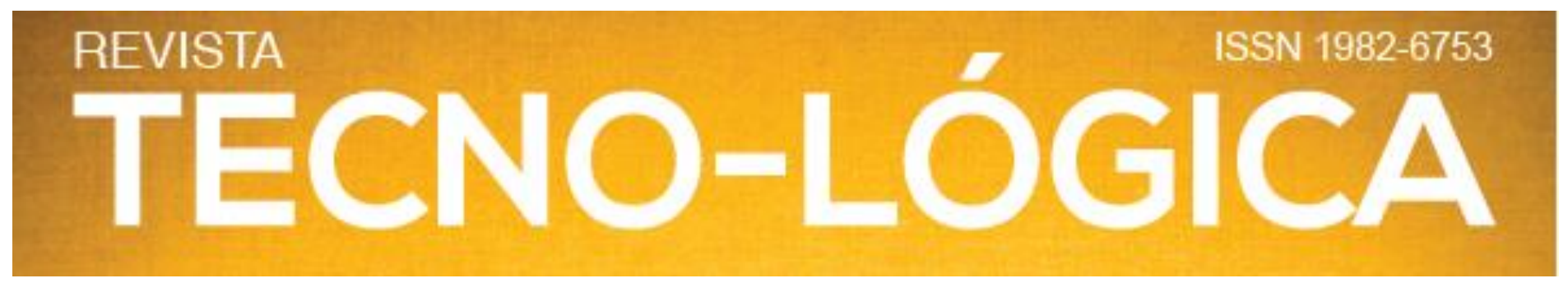

Ademais, para que um comando raramente utilizado possa ser aceito fora da zona de conforto, as forças de manobra não devem ser superiores às admitidas para os comandos frequentemente utilizados. Entretanto, a norma ISO 15077 [13] não esclarece quais são os comandos que jamais podem ser posicionados fora da zona de conforto. Assim, com base nas especificações estabelecidas pela norma ISO 15077 [13], os comandos analisados foram agrupados de acordo com sua frequência de uso. Os dados coletados foram submetidos a uma análise exploratória por meio de estatística descritiva com uso de frequência percentual.

\section{Resultados e discussões}

A distribuição dos comandos das duas colhedoras de grãos avaliadas, em relação ao plano longitudinal vertical está representada na Figura 4.
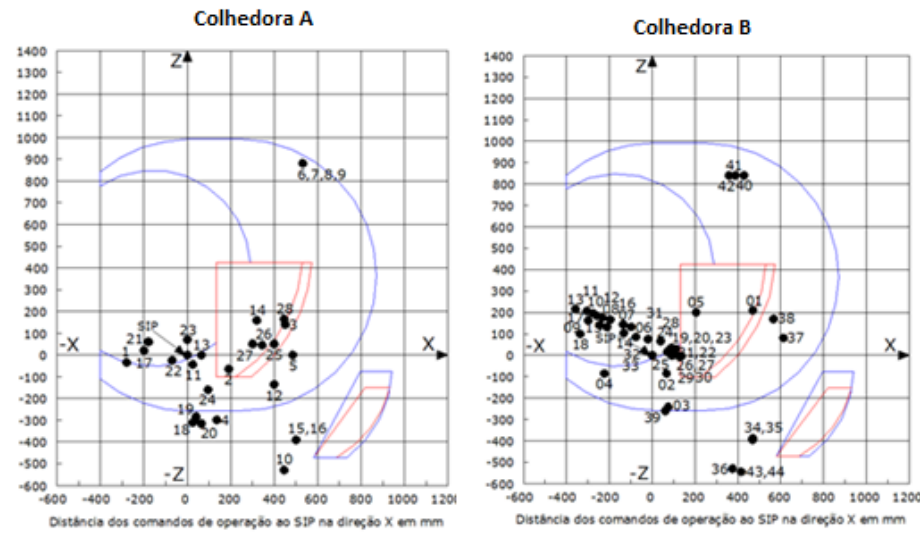

Figura 4 - Distribuição dos comandos de operação no plano longitudinal vertical das colhedoras de grãos A e B.

Para o plano longitudinal vertical, considerando os comandos de acionamento frequente (Figura 5A), a colhedora A apresentou $25 \%$ de seus comandos situados na zona de conforto, $41,67 \%$ na zona de acesso e 33,30\% na zona inacessível. Ao analisar da mesma forma, a colhedora B apresentou apenas 2,7\% dos comandos localizados na zona de conforto, $54,05 \%$ na zona de acesso e $43,24 \%$ na zona inacessível.

Ao analisar o plano longitudinal vertical, considerando os comandos de acionamento raro (Figura 5B), a colhedora A possui $38 \%$ de seus 41 comandos situados na zona de conforto, $45,83 \%$ na zona de acesso e $16,67 \%$ dos comandos restantes localizados na zona inacessível. Já a colhedora B, apresentou 21,62\% dos comandos localizados na zona de conforto, $70,27 \%$ na zona de acesso e apenas $8,11 \%$ na zona inacessível.
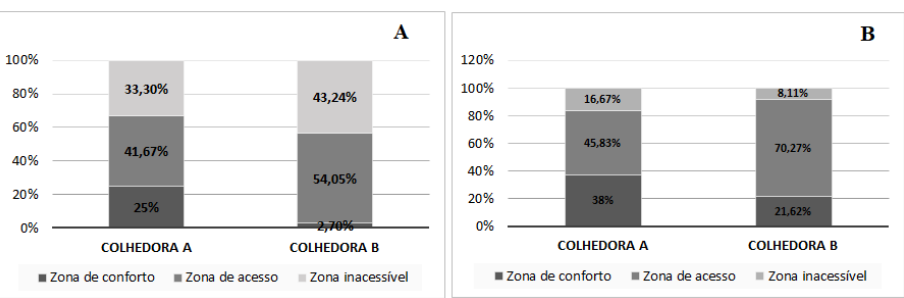

Figura 5 - Comandos de acionamento frequente (A) e raro (B) no plano longitudinal vertical.

Ao considerar os comandos de acionamento frequente em relação ao plano longitudinal vertical, a colhedora A apresentou $70,67 \%$ de seus comandos dentro das zonas de conforto e de acesso, enquanto a colhedora B 56,75\% nestas duas zonas, demostrando condições ergonômicas diferentes entre os modelos estudados. A variação das condições ergonômicas entre diferentes modelos de máquinas agrícolas também foi descrita por Rozin et al. [6] que, ao analisarem o posicionamento dos comandos de operação de tratores agrícolas, observaram variações consideráveis entre modelos e fabricantes.

Além disso, outro fator referente aos comandos de acionamento frequente que deve ser considerado no projeto de colhedoras de grãos são as características antropométricas do operador. Estas características variam entre países e regiões e muitas vezes não são contempladas pelas normas. Corroborando com o exposto, Santos et al. [15] em estudo sobre o perfil antropométrico de operadores de tratores no Nordeste brasileiro, afirmam que as normas ISO e NBR utilizadas são inadequadas ou parcialmente inadequadas para os operadores avaliados, nas condições em que o estudo foi realizado.

Por isso, a observação da posição dos comandos de operação dentro das zonas de conforto e de acesso é indispensável na adequação de novos projetos por parte dos fabricantes. Faz-se necessário considerar as características do operador e realizar o trabalho buscando alcançar maior eficiência e produtividade, com conforto e segurança [4]. Em um estudo realizado por Simões et al. [1], ao analisarem ergonomicamente três modelos de colhedoras de cana-de-açúcar, os pesquisadores mencionam que as máquinas não apresentavam indicadores de função facilmente compreendidos pelo operador, bem como a posição e a distância dos comandos de operação, mesmo assim, a posição do operador não foi prejudicada.

Com relação à distribuição dos comandos referentes ao plano horizontal superior (Figura 6), pode-se observar variações 


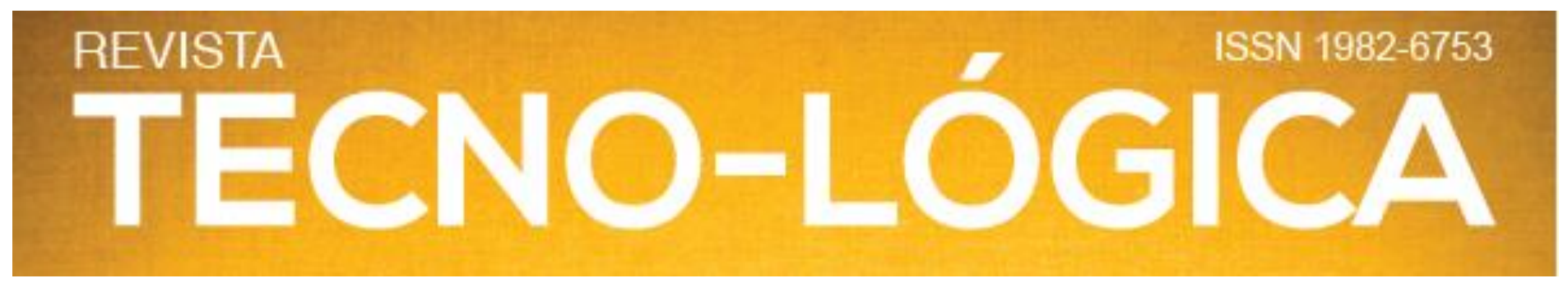

entre as duas colhedoras estudadas, considerando tanto os comandos de acionamento frequente, quanto os de acionamento raro.
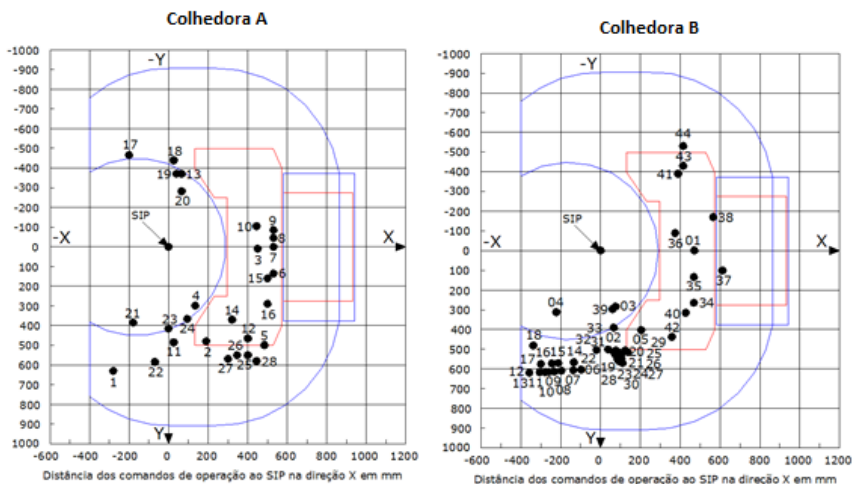

Figura 6 - Distribuição dos comandos no plano horizontal superior das colhedoras A e B.

Para o plano horizontal superior, considerando os comandos de acionamento frequente (Figura $7 \mathrm{~A}$ ), a colhedora A apresentou $25 \%$ de seus comandos situados na zona de conforto e $75 \%$ localizados na zona de acesso. Já a colhedora B apresentou $28,57 \%$ dos comandos localizados na zona de conforto, $42,86 \%$ na zona de acesso e $28,57 \%$ na zona inacessível.

Considerando os comandos de acionamento raro referente ao plano horizontal superior (Figura 7B), a colhedora A apresentou $75 \%$ dos comandos situados na zona de conforto e $25 \%$ na zona de acesso. A colhedora B apresentou 57,14\% dos comandos localizados na zona de conforto e $42,86 \%$ na zona de acesso.

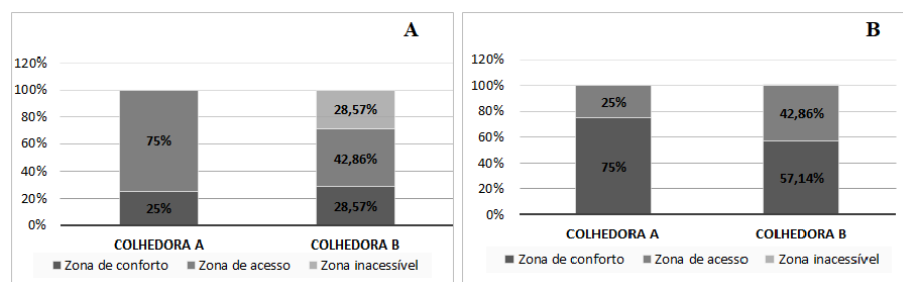

Figura 7 - Comandos de acionamento frequente (A) e raro (B) no plano horizontal superior.

Cabe ressaltar que, no plano horizontal superior, ocorre o maior número de repetições e movimentos de acionamento dos comandos. A colhedora A apresentou melhores condições ergonômicas, tendo todos os comandos de acionamento frequente dentro das zonas de conforto e de acesso, assim como os comandos de acionamento raro, embora a colhedora $\mathrm{B}$ tenha o maior número de comandos de acionamento frequente dentro da zona de conforto. Com relação à colhedora B, pode-se afirmar que 71,43\% dos comandos de acionamento frequente encontram-se nas zonas de conforto e de acesso e na totalidade os comandos de acionamento raro.

Em relação aos planos longitudinal vertical e horizontal superior analisados e relacionados às zonas de conforto, de acesso e inacessível, observaram-se variações consideráveis no posicionamento dos comandos nos dois modelos de colhedoras de grãos, demonstrando que os fabricantes obedecem diferentes padrões construtivos.

Corroborando com os resultados encontrados, Minette et al. [16] destacam que todas as máquinas apresentam alguns de seus comandos fora dos números considerados adequados. Rozin et al. [6] mencionam que os projetistas de máquinas devem repensar o ambiente de trabalho adequado ao operador, podendo ser variável entre diferentes países. No entanto, devido ao atual desenvolvimento da agricultura, a indústria de máquinas tem observado o posto do operador com relação aos parâmetros ergonômicos, porém, vários requisitos ainda são negligenciados [17].

\section{Conclusões}

A disposição dos comandos de acionamento frequente e raro, referentes aos planos longitudinal vertical e horizontal superior, apresentou variação quantitativa e qualitativa considerável para os modelos de colhedoras estudados.

A colhedora A apresentou melhores condições ergonômicas, contemplando mais da metade dos comandos de acionamento frequentes situados nas zonas de conforto e de acesso, para ambos os planos analisados

\section{ERGONOMIC EVALUATION OF THE OPERATOR'S WORKPLACE OF TWO COMBINE HARVESTERS}

ABSTRACT: Ergonomic conditions related to the operator's workplace have been decisive for the development of new agricultural machinery projects, providing adequate conditions for operators and efficiency during the performance of operations. In this sense, this work aimed to evaluate the ergonomic characteristics of the operator's workplace of two combine harvesters, related to the access zones and the operation commands. Two combine harvesters, named A and B, belonging to Class IV, were studied, and the positioning of the main operating commands related to the Seat Index Point, classified in access zones, according to the ISO 15077 standard, was evaluated. The results indicate that, $70.67 \%$ and $56.75 \%$ of the frequently activated controls are located in the comfort and access zones in 
the vertical longitudinal plane, with $100 \%$ and $71.43 \%$ of the commands related to the upper horizontal plane in these areas, for harvesters $\mathrm{A}$ and $\mathrm{B}$, respectively. Therefore, there was a considerable variation in the arrangement of the drive controls, for the vertical longitudinal and horizontal upper planes, for both models of harvesters studied.

Keywords: Agricultural mechanization. Operating controls. Ergonomics.

\section{Referências}

[1] SIMÕES, D. et al. Ergonomic characterization of three sugar cane harvester machinery models. African Journal of Agricultural Research, v. 11, p. 724-729, 2016.

[2] MAZETTO, F. R. Avaliação dos desempenhos operacional e energético e da ergonomia de colhedoras de soja (Glycine max (L.) Merril) no sistema de plantio direto. 2008. $104 \mathrm{f}$. Tese (Doutorado em Energia na Agricultura) - Universidade Estadual Paulista, Botucatu, SP

[3] IIDA, I. Ergonomia: projeto e produção. $2^{\mathrm{a}}$ ed. São Paulo: Edgard Blücher, 2005. 465p.

[4] FONTANA, G.; SEIXAS, F. Avaliação ergonômica do posto de trabalho de modelos de forwarder e skidder. Árvore, v. 31, n. 1, p. 71-81, 2007.

[5] ANDRADE, P. A. M. Avaliação de parâmetros ergonômicos: ruído, temperatura e iluminação no posto operacional de tratores agrícolas. 2017. $96 \mathrm{f}$. Dissertação (Mestrado em Energia da Agricultura) - Universidade Estadual Paulista, Botucatu, SP.

[6] ROZIN, D. et al. Conformidade dos comandos de operação de tratores agrícolas nacionais com a norma NBR ISO 4253. Revista Brasileira de Engenharia Agrícola e Ambiental, v. 14, n. 9, p. 1014-1019, 2010

[7] BARBIERI, J. P. et al. The development and validation of an ergonomics index for assessing tractor operator work place. Ciência Rural, v. 48, n. 1, p.1-7, 2018.

[8] DUL, J.; WEERDMEESTER, B. Ergonomia Prática. $2^{\text {a }}$ ed. São Paulo: Ed. Edgard Blücher, 2004. 137p.

[9] CASALI, A. L. et al. Conformidade do posto de operação de pulverizadores autopropelidos. Engenharia na Agricultura, v. 19, n. 6, p. 548-556, 2011.

[10] POSSEBON, G. Comparação de métodos para avaliação postural em operação de máquinas agrícolas. 2018. 103 f. Dissertação (Mestrado em Engenharia Agrícola) - Universidade Federal de Santa Maria, Santa Maria, RS.

[11] PERIPOLLI, J. L. Z. et al. Conformidade do espaço interno livre de tratores agrícolas e itens de segurança obrigatórios segundo as normas NBR/ISO 4252 e NR-12. Tecno-Lógica, v. 21, n. 2, p. 103-107, 2017.
[12] INTERNATIONAL ORGANIZATION FOR STANDARDIZATION. Earth moving machinery - Seat Index Point: ISO 5353. Genève, 1999.

[13] Tractors and machinery for agriculture and forestry - operator controls Actuating forces, their displacement and location: ISO 15077. Genève, 1996.

[14] Agricultural Tractors - Operator's seating accommodation - Dimensions: ISO 4253. Genève, 1993.

[15] SANTOS, V. C. et al. Tractor operator anthropometric profile of the Brazilian Northeast State. African Journal of Agricultural Research, v. 11, p. 4850-4856, 2016

[16] MINETTE, L. J.; SOUZA, A. P. de; SILVA, E. P.; MEDEIROS, N. M. Postos de trabalho e perfil de operadores de máquinas de colheita florestal. Revista Ceres, v. 55, n. 1 , p. $66-73,2008$.

[17] NIETIEDT G. H. et al. Distribuição dos comandos de operação em tratores agrícolas nacionais com até $55 \mathrm{~kW}$ de potência. Revista Brasileira de Engenharia Agrícola e Ambiental, v. 16, n. 6, p. 690-695, 2012. 